\title{
Burden in Family Caregivers of Cancer Patients: The Association with Depression, Religiosity and Religious Coping
}

\author{
Vun Kong Shim 1 , Chong Guan $\mathrm{Ng}^{2}$
}

${ }^{1}$ Hospital Sentosa, Kuching, Sarawak, Malaysia. ${ }^{2}$ Department of Psychological Medicine, Universiti Malaya Medical Centre, Kuala Lumpur, Malaysia.

\begin{abstract}
Introduction: Cancer caregiving is challenging and often associated with significant burden in family caregivers. Yet, the healthcare providers often overlook the needs of the caregivers. Local data on cancer caregiving is still lacking. In line with the increasing cancer cases worldwide, there is a need to examine factors influencing the caregiver burden, and to find ways to improve the quality of life of the caregivers. Objectives: The study aimed to determine the caregiver burden rate among the local family caregivers of cancer patients, and to examine the associations between the caregiver burden and their sociodemographic factors, clinical factors, levels of depression, religiosity, and religious coping patterns. Methods: 127 family caregivers of cancer patients in a government tertiary hospital in Sarawak, Malaysia were recruited cross-sectionally using non-random sampling method. The associations between the variables were examined through bivariate analyses (Chi-Square test), followed by multivariate analysis to determine the factor (s) that were significantly associated with the caregiver burden. Results: The caregiver burden rate among local cancer caregivers was $55.6 \%$. Two factors remained significant after adjusted in multivariate analysis. Caregivers who were the offspring of cancer patients experienced significantly fewer burden than non-offspring caregivers (OR 0.41, CI $0.18-0.94, \mathrm{p}=0.035$ ). Also, caregiver depression was significantly associated with caregiver burden (OR 4.26, CI $1.87-9.72, \mathrm{p}=0.001)$. There were no significant associations found between religiosity and religious coping with the caregiver burden on multivariate level. Conclusion: Caregiver burden is common among family caregivers of cancer patients. Caregivers who are the children or grandchildren of cancer patients are less likely to experience caregiver burden, whereas caregivers who have probable depression are more likely to experience caregiver burden. Future studies should assess the effects of specific intervention strategies in helping these caregivers.
\end{abstract}

Keywords: Family caregiver- cancer- burden- depression- religiosity- religious coping

Asian Pac J Cancer Care, 4 (4), 171-182

\section{Introduction}

Cancer is one of the leading causes of morbidity and mortality worldwide. In 2018, approximately 18 million new cases were diagnosed based on the Global Burden of Cancer study conducted by the International Agency for Research on Cancer of the World Health Organization. This number is postulated to increase to over 20 million by 2025 , with the low and middle-income countries bearing most of the cancer burden [1]. According to the Malaysian National Cancer Registry, a total of 103,507 new cancer cases were diagnosed between 2007 and 2011 in Malaysia. Sarawak state documented 9,734 new cancer cases or equivalent to about $9.4 \%$ of the national figures [2].
Submission Date: 04/20/2019 Acceptance Date: 07/26/2019

It is well known that cancer causes significant physical and emotional impairments in its sufferers [3-4]. Not only the patients, cancer also indirectly affects their significant others, many of whom are the primary caregivers [5-6]. Cancer patients and their caregivers are frequently exposed to the complications of the disease itself.

According to Family Caregiver Alliance based in the United States, family (or informal) caregiver refers to any person, e.g. spouse, adult children, other relatives, neighbour or friend, who has a personal relationship with, and provides a wide range of unpaid assistance for, an older person or an adult with a chronic or disabling condition [7]. This is different from a professional (or formal) caregiver, who is either a paid carer or a volunteer with no personal

Corresponding Author:

Dr. Vun Kong Shim

Hospital Sentosa, Kuching, Sarawak, Malaysia.

Email: shimken83@gmail.com 
relationship with the person he or she is looking after.

There is a significant reciprocal relationship between the emotional distress of cancer patients and their caregivers [8-9]. Thus, the management of cancer patients would be compromised if the caregivers' well-being is affected [10]. Despite caregiving has a significant impact on the caregivers' well-being, the needs of the caregivers are often overlooked or considered secondary to those of the patients [11].

Recent advancement in the diagnostic and therapeutic modalities has resulted in a paradigm shift in the cancer course and care plan [12]. The course of cancer has changed from being an acute condition with rapid or direct consequences, usually fatality, to a chronic illness with variable outcomes [13]. This may translate to a need for long-term and continuous care for cancer patients with the integration of both informal and formal care systems.

Consequently, family caregivers are at increased risk of being burdened with multiple stressful physical and psychosocial problems [14]. Psychological problems such as anxiety, depression, and loneliness, are the commonly recognized burdens on caregivers [15]. In line with the increasing trend of cancer cases and caregiver burden worldwide, there is a need to look into the caregiving aspects and to find ways to improve the well-being of the family caregivers.

One suggested the solution is through the study of religious or spiritual beliefs and practices. Yet, research on the roles of religion and its relation to the human well-being accounts only a small fraction of the current literature in psychology [16]. Religiosity, spirituality and religious coping have been shown to play important buffering roles in helping cancer patients to deal with psychological distress [17]. Thus, these benefits might be extended to cancer caregivers as well.

There were many studies done overseas on the subject of caregiver burden among the family caregivers of cancer patients [9-13-18]. However, most of the studies only identified the caregiver burden in general but did not specifically look at the factors associated with the high burden among the caregivers [19]. In Malaysia, local published data about this subject is still lacking despite the increasing trend of cancer burden nationwide.

As to date, majority of the related studies on cancer caregiver burden in Malaysia were conducted in Peninsular Malaysia [10-20], with only one qualitative study being carried out in Sarawak thus far [21]. The Sarawak study explored the caregiving experience of nine Chinese cancer caregivers and implied the roles of nursing support in easing the suffering of the caregivers [21]. However, the study did not determine the rate of caregiver burden and look into the various factors affecting this burden.

A better understanding of these factors is crucial to shed light on previously unrecognized issues that may affect caregiving outcomes. Hopefully, the findings from this study can guide the policymakers and the healthcare professionals on how to implement targeted intervention strategies to help the family caregivers in the future. Besides, this preliminary study might provide some direction for future research in this area relevant to the local setting.

\section{Materials and Methods}

\section{Study Design and Setting}

This study was a cross-sectional study on the burden of the cancer caregivers; and its association with caregiver depression, religiosity, and religious coping styles. The source population was the family caregivers of any cancer patients, either outpatients or inpatients, at the Department of Radiotherapy, Oncology and Palliative Care of Sarawak General Hospital (SGH) located in Kuching, Sarawak, Malaysia. SGH functions as a tertiary center for the referral and treatment of cancer cases from the whole state of Sarawak. Data for this study were collected from January 2017 until April 2017.

\section{Study Criteria}

Inclusion criteria: (1) Family caregivers aged 18 years and above, (2) Identified by cancer patients as primary caregiver, (3) Able to understand the Malay language, (4) Able to give informed consent to participate in the study. Exclusion criteria: (1) Paid, professional or formal caregivers, (2) Caregivers who were diagnosed with any pre-existing major psychiatric disorder based on DSM-5 criteria.

\section{Study Procedure}

This study represented the main phase of a two-phased cross-sectional study. The first phase was conducted to validate the Malay version of the Zarit Burden Interview (MZBI) scale [22]. The main phase aimed to determine the caregiver burden rate among the local family caregivers of cancer patients and to examine the associations between the caregiver burden and their sociodemographic factors, clinical factors, levels of depression, religiosity, and religious coping patterns.

A single investigator carried out the data collection. The sample was collected from the family caregivers that were conveniently available at the study site. Participation was based voluntarily. The subjects were given ample time to read and understand the study information sheet. All data were collected independently with full confidentiality assured.

\section{Study Measures}

\section{Sociodemographic and Clinical Questionnaire}

A single investigator interviewed the subjects to ensure uniformity of the data collected. As far as possible, the information was obtained from the caregivers directly. In cases where there was the ambiguity of the information obtained, patients' medical record was referred for confirmation.

\section{Malay version of Zarit Burden Interview (MZBI)}

Zarit Burden Interview (ZBI) is the most widely used measure for caregiver burden assessment [23]. This self-administered, validated, 22-item scale evaluates caregiver's physical health, psychological and social 
well-being, finances, and the relationship between caregiver and care recipient [24]. In this measure, each item consists of a question in which the caregiver is asked to respond using a 5-point Likert scale. The total score ranges from 0 to 88 , with higher scores indicating greater caregiver burden. The MZBI demonstrated high internal consistency (Cronbach's alpha 0.898) and split-half correlation of 0.912 . A score of 22 was selected as the suitable cut-off score in a local setting with a sensitivity of $70.8 \%$ and a specificity of $69.2 \%$ [22].

\section{Malay version of Center for Epidemiological Studies - Depression (MCES-D)}

Center for Epidemiological Studies - Depression (CES-D) scale was commonly used as a screening tool in epidemiological studies of depression [25]. The measure consists of 20 items rated on a 4-point Likert scale to indicate how often the respondent felt that way over the previous week. The total score ranges from 0 to 60 . A cut-off score of 16 is suggested for identification of potential clinical depression in the respondents [25]. Validation study of MCES-D among a group of Malaysian junior doctors reported good internal consistency (Cronbach's alpha 0.78) [26].

\section{Malay version of Duke University Religion Index (DUREL-M)}

The Duke University Religion Index (DUREL) is designed to measure the religious commitment of the respondent [27-28]. It consists of five items covering three major dimensions of religiosity: organizational religious activity (ORA, 1 item); non-organizational religious activity (NORA, 1 item); and intrinsic religiosity (IR, 3 items). ORA and NORA are scored from 1 to 6 , whereas the three items in IR are scored from 1 to 5 . The original authors recommended the measure to be interpreted separately within the three subscales of ORA, NORA, and IR, corresponding to the three distinct religious dimensions measured by them [27]. The Malay version of the scale (DUREL-M) was validated among a group of Malaysian nursing students with good Cronbach's alpha of 0.80 and fair test-retest reliability (Spearman's correlation 0.68, $\mathrm{p}<0.01$ ) [29].

\section{Malay version of Brief Religious Coping Questionnaire (M-RCOPE)}

The Brief Religious Coping Questionnaire (Brief RCOPE) is the concise version of the original RCOPE developed based on Pargament's theory on religious coping [30]. It consists of 14 items: seven Positive Religious Coping (PRC) items and seven Negative Religious Coping (NRC) items used to measure the religious coping pattern of the respondent [30].

The scoring for each of the 14 items ranges from 1 to 4 . The total score ranges from 7 to 28 for each of the subscales. The Brief RCOPE was translated into the Malay language (M-RCOPE) and validated in 68 Malaysian women with breast cancer [31]. The study showed good internal consistencies for the two domains in M-RCOPE (Cronbach's alpha 0.87 for PRC and 0.88 for NRC).

\section{Statistical Analysis}

All statistical analyses were performed using Statistical Package for the Social Sciences (IBM SPSS) version 24. Median and interquartile range (IqR) were used to describe the continuous variables, as the data were not normally distributed in the non-randomized sampling design. Values for mean and standard deviation were also displayed for comparison. Bivariate analyses using Chi-Square test for categorical data were carried out to determine the association between caregiver burden with all the other variables. Multiple logistic regression analysis was then performed on the significant factors from the bivariate analyses. A p-value of less than 0.05 (two-sided) with a $95 \%$ confidence interval was considered as statistically significant for all analyses. For incomplete datasets, the missing values were excluded but the remaining data were still being included in the analyses.

\section{Ethical Approval}

This study was registered under the National Medical Research Registry (NMRR). Ethical approval was obtained from the Medical Research and Ethics Committee (MREC) of the Ministry of Health Malaysia.

\section{Results}

\section{Sociodemographic Characteristics}

The median age of the 127 family caregivers was 42 years old (range $19-67$ years, IqR $=18$ years). Female caregivers $(67.7 \%)$ were twice the number of male caregivers. Bumiputera Sarawak (56.7\%) constituted the majority, followed by Malay $(25.2 \%)$ and Chinese (15.7\%) caregivers. Majority of the Bumiputera Sarawak caregivers were Ibans $(n=39)$ and Bidayuhs $(n=20)$. The commonest religion was Christianity (55.1\%), followed by Islam (34.6\%). Three of the caregivers $(2.4 \%)$ had no religion.

Majority of the caregivers were married $(80.3 \%)$, unemployed $(52 \%)$, and from the low-income group with household income less than RM 3,000 per month (56.7\%). A substantial portion of the caregivers was well educated with $63.8 \%$ had secondary education and $24.4 \%$ had tertiary education. The majority were the spouses of the cancer patients $(44.9 \%)$, followed by the children or grandchildren $(41.0 \%)$ and the parents $(6.3 \%)$. Table 1 summarizes the sociodemographic characteristics of the study subjects.

\section{Clinical Characteristics}

Majority of the caregivers were looking after relatives with solid cancer $(92.9 \%)$, with the rest being hematological malignancies. Nasopharyngeal cancer $(\mathrm{n}=27)$ was the commonest solid cancer, followed by colorectal $(n=23)$ and breast $(n=22)$ cancers. As for hematological malignancies $(n=9)$, five had leukemia, three had lymphoma and one had aplastic anemia. In terms of cancer stages, $42.5 \%$ were of moderate severity, followed by severe cancer or Stage 4 (32.3\%). 
Table 1. Sociodemographic Characteristics of Family Caregivers $(\mathrm{N}=127)$

\begin{tabular}{|c|c|c|c|}
\hline Characteristics & $\mathrm{n}(\%)$ & Mean \pm SD & Median (IqR) \\
\hline Age (years) & & $41.1 \pm 12.27$ & $42.0(18.0)$ \\
\hline$<42$ & $61(48.0)$ & & \\
\hline$\geq 42$ & $66(52.0)$ & & \\
\hline \multicolumn{4}{|l|}{ Gender } \\
\hline Male & $41(32.3)$ & & \\
\hline Female & $86(67.7)$ & & \\
\hline \multicolumn{4}{|l|}{ Ethnicity } \\
\hline Malay & $32(25.2)$ & & \\
\hline Chinese & $20(15.7)$ & & \\
\hline Bumiputera Sarawak & $72(56.7)$ & & \\
\hline Others & $3(2.4)$ & & \\
\hline \multicolumn{4}{|l|}{ Marital status } \\
\hline Single & $25(19.7)$ & & \\
\hline Married & $102(80.3)$ & & \\
\hline \multicolumn{4}{|l|}{ Religion } \\
\hline Christian & $70(55.1)$ & & \\
\hline Muslim & $44(34.6)$ & & \\
\hline Buddhist & $9(7.1)$ & & \\
\hline Others & $1(0.8)$ & & \\
\hline No religion & $3(2.4)$ & & \\
\hline \multicolumn{4}{|l|}{ Employment } \\
\hline Yes & $61(48.0)$ & & \\
\hline No & $66(52.0)$ & & \\
\hline \multicolumn{4}{|l|}{ Household income (per month) } \\
\hline$<\mathrm{RM} 3,000$ & $72(56.7)$ & & \\
\hline Between RM3,000 to RM10,000 & $41(32.3)$ & & \\
\hline$\geq \mathrm{RM} 10,000$ & $14(11.0)$ & & \\
\hline \multicolumn{4}{|l|}{ Education level } \\
\hline Tertiary & $31(24.4)$ & & \\
\hline Secondary & $81(63.8)$ & & \\
\hline Primary & $15(11.8)$ & & \\
\hline \multicolumn{4}{|l|}{ Relationship to relative with cancer } \\
\hline Spouses & $57(44.9)$ & & \\
\hline Children/Grandchildren & $52(41.0)$ & & \\
\hline Parents & $8(6.3)$ & & \\
\hline Siblings & $6(4.7)$ & & \\
\hline Others & $4(3.1)$ & & \\
\hline
\end{tabular}

IqR, Interquartile range; RM, Ringgit Malaysia; SD, Standard deviation

Majority of the relatives were receiving treatment as inpatient $(56.7 \%)$. Most of them were on active treatment $(73.2 \%)$, while only ten $(7.9 \%)$ were on palliative care.

The functional status of the relatives was assessed using the Eastern Cooperative Oncology Group (ECOG) scoring [32]. Score 0 and 1 correspond to relatives that were still able to work or perform house chores, whereas a score of 2 or less indicates the relatives were still capable of caring for themselves. In this study, slightly more than half of the cancer patients were not able to work or do house chores $(52.8 \%)$, but most were still capable of self-care (85\%).

Looking at the caregiving aspects, the majority of the caregivers have shared caregiving responsibilities with other family caregivers $(78.7 \%)$, with the average having two other caregivers assisting in the care of their relatives. The median duration as a caregiver in this study was nine months (range $1-156$ months, IqR 20 months). As for the total time spent on caregiving in a week, the median time was 128 hours (range $2-168$ hours, IqR 120 hours). Table 2 summarizes the clinical characteristics of the subjects. 
Table 2. Clinical Characteristics of Family Caregivers $(\mathrm{N}=127)$

\begin{tabular}{|c|c|c|c|}
\hline Characteristics & $\mathrm{n}(\%)$ & Mean \pm SD & Median (IqR) \\
\hline \multicolumn{4}{|l|}{ Types of primary cancer in relative } \\
\hline Solid & $118(92.9)$ & & \\
\hline Hematological & $9(7.1)$ & & \\
\hline \multicolumn{4}{|l|}{ Stages of cancer in relative } \\
\hline Stage 1/Mild & $12(9.5)$ & & \\
\hline Stage 2-3/Moderate & $54(42.5)$ & & \\
\hline Stage $4 /$ Severe & $41(32.3)$ & & \\
\hline Unsure stage & $20(15.7)$ & & \\
\hline \multicolumn{4}{|l|}{ Treatment setting of relative } \\
\hline In-patient & $72(56.7)$ & & \\
\hline Out-patient & $55(43.3)$ & & \\
\hline \multicolumn{4}{|l|}{ Treatment types received by relative } \\
\hline Active treatment & $93(73.2)$ & & \\
\hline Palliative treatment & $10(7.9)$ & & \\
\hline Not yet plan for treatment & $15(11.8)$ & & \\
\hline Completed treatment & $9(7.1)$ & & \\
\hline \multicolumn{4}{|l|}{ Functional status of relative (based on ECOG score) } \\
\hline Score 0 & $5(3.9)$ & & \\
\hline Score 1 & $55(43.3)$ & & \\
\hline Score 2 & $48(37.8)$ & & \\
\hline Score 3 & $13(10.3)$ & & \\
\hline Score 4 & $6(4.7)$ & & \\
\hline \multicolumn{4}{|l|}{ Relative still able to work/do chores } \\
\hline Yes & $60(47.2)$ & & \\
\hline No & $67(52.8)$ & & \\
\hline \multicolumn{4}{|l|}{ Relative capable of self-care } \\
\hline Yes & $108(85.0)$ & & \\
\hline No & $19(15.0)$ & & \\
\hline \multicolumn{4}{|l|}{ Shared caregiving } \\
\hline Yes & $100(78.7)$ & & \\
\hline No & $27(21.3)$ & & \\
\hline Duration as a caregiver (months) & & $17.3 \pm 22.60$ & $9.0(20.0)$ \\
\hline Time spent on caregiving in a week (hours) & & $109.9 \pm 60.75$ & $128.0(120.0)$ \\
\hline Number of other family caregivers assisting in caregiving & & $2.0 \pm 1.67$ & $2.0(2.0)$ \\
\hline
\end{tabular}

ECOG, Eastern Cooperative Oncology Group; IqR, Interquartile range; SD, Standard deviation

Descriptive Statistics for MZBI, MCES-D, DUREL-M and M-RCOPE Scores

The median score for MZBI based on the responses gathered from the subjects was 26.0 (range $0-67$, interquartile range 20.25). The mean scores of the individual items in the MZBI ranged from 0.4 to 2.8 . All individual item scores were significantly correlated to the total MZBI score through Spearman's correlation $(\mathrm{p}<0.01)$. Based on the MZBI cut-off score of 22 , majority of the family caregivers $(55.6 \%)$ were found to have significant caregiver burden.

The scores for MCES-D ranged from 0 to 47, with a median score of 19 (interquartile range 17). Based on the cut-off score of 16 for MCES-D scale, more than half of the 127 caregivers (59.1\%) in this study were having probable depression.

For DUREL-M, the total scores in this study ranged from 7 to 27 with a median score of 23 (interquartile range 5). The study subjects were divided into two groups: those who scored 23 and above the median score (high religiosity), and those who scored below the median score (low religiosity). The group with high religiosity (56.7\%) constituted the majority.

The M-RCOPE was interpreted separately for two of its main subscales. For PRC, the median score was 25 (interquartile range 7) within a range of scores from 7 to 28. Comparatively, the median score for NRC was lower (median 10, interquartile range 5) with scores ranged from 7 to 27 . Similarly, the study subjects were divided into two groups based on the median scores for 
Table 3. Bivariate Analysis of the Association between Caregiver Burden with Sociodemographic Characteristics among the Study Subjects using Chi-square Test $(\mathrm{N}=126)$

\begin{tabular}{|c|c|c|c|c|c|c|}
\hline \multirow{3}{*}{ Characteristics } & \multicolumn{2}{|c|}{ Caregiver Burden $^{\text {a }}$} & \multirow[t]{3}{*}{$\chi^{2}$} & \multirow[t]{3}{*}{$\mathrm{OR}$} & \multirow[t]{3}{*}{$95 \% \mathrm{CI}$} & \multirow[t]{3}{*}{ p-value } \\
\hline & No & Yes & & & & \\
\hline & $\mathrm{n}(\%)$ & $\mathrm{n}(\%)$ & & & & \\
\hline Age (years) & & & 2.420 & 1.75 & $0.86-3.57$ & 0.120 \\
\hline$<42$ & $31(51.7)$ & $29(48.3)$ & & & & \\
\hline$\geq 42$ & $25(37.9)$ & $41(62.1)$ & & & & \\
\hline Gender & & & 3.386 & 2.08 & $0.95-4.55$ & 0.066 \\
\hline Female & $43(50.0)$ & $43(50.0)$ & & & & \\
\hline Male & $13(32.5)$ & $27(67.5)$ & & & & \\
\hline Ethnicity & & & 1.646 & 0.54 & $0.21-1.40$ & 0.200 \\
\hline Bumiputera & $44(41.9)$ & $61(58.1)$ & & & & \\
\hline Non-Bumiputera & $12(57.1)$ & $9(42.9)$ & & & & \\
\hline Marital status & & & 3.056 & 2.20 & $0.90-5.36$ & 0.080 \\
\hline Single/Divorced/Widowed & $15(60.0)$ & $10(40.0)$ & & & & \\
\hline Married & $41(40.6)$ & $60(59.4)$ & & & & \\
\hline Employment & & & 0.229 & 1.19 & $0.59-2.40$ & 0.632 \\
\hline Yes & $28(46.7)$ & $32(53.3)$ & & & & \\
\hline No & $28(42.4)$ & $38(57.6)$ & & & & \\
\hline Household income & & & 0.316 & 0.82 & $0.40-1.66$ & 0.574 \\
\hline$<$ RM3000 per month & $30(42.3)$ & $41(57.7)$ & & & & \\
\hline$\geq$ RM3000 per month & $26(47.3)$ & $29(52.7)$ & & & & \\
\hline Education level & & & 0.105 & 0.87 & $0.39-1.98$ & 0.746 \\
\hline Tertiary & $13(41.9)$ & $18(58.1)$ & & & & \\
\hline Primary /Secondary & $43(45.3)$ & $52(54.7)$ & & & & \\
\hline Relationship to cancer patient & & & 1.969 & 1.67 & $0.82-3.41$ & 0.161 \\
\hline Non-spouses & $35(50.0)$ & $35(50.0)$ & & & & \\
\hline Spouses & $21(37.5)$ & $35(62.5)$ & & & & \\
\hline Relationship to cancer patient & & & 4.599 & 0.46 & $0.22-0.94$ & $0.032 *$ \\
\hline Non-children/grandchildren & $27(36.5)$ & $47(63.5)$ & & & & \\
\hline Children/grandchildren & $29(55.8)$ & $23(44.2)$ & & & & \\
\hline
\end{tabular}

$\chi^{2}$, Chi-square; OR, Odds ratio; CI, Confidence interval; * Statistically significant $(\mathrm{p}<0.05)$; Presence of caregiver burden was based on the total MZBI score of equal or more than 22 .

PRC and NRC respectively: High PRC group (scored 25 and above) versus low PRC group (scored below 25); and high NRC group (scored 10 and above), versus low NRC group (scored below 10). Both high PRC group (53.5\%) and high NRC group (57.5\%) were more in numbers.

\section{Association between Caregiver Burden and Sociodemographic Characteristics}

Bivariate analysis was performed to examine the associations between caregiver burden and caregivers' sociodemographic factors (Table 3). One factor was found to be significantly associated with caregiver burden: Relationship to cancer patients being the offspring. In other words, caregivers who were the children or grandchildren of the cancer patients were significantly less likely to have caregiver burden than caregivers who were not (OR 0.46, CI $0.22-0.94, \mathrm{p}=0.032$ ).
Association between Caregiver Burden and Clinical Characteristics

Clinical characteristics in relatives of the caregivers such as types of primary cancer, severity of cancer, treatment settings, treatment types, and functional status (ability to work and capacity for self-care); as well as caregiving factors such as presence of shared caregiving, external support, and caregiving duration were examined for associations with the caregiver burden (Table 4). Two factors were found to be significant: Treatment setting and the ability to work. Significant caregiver burden was more likely among family caregivers who cared for relatives that were treated as inpatients than those that were treated as outpatients (OR 2.21, CI $1.08-4.55, \mathrm{p}=0.03$ ). Also, caregivers who looked after cancer patients that were unable to work experienced significantly more burden than those who cared for relatives that were still able to work (OR 2.43 CI 1.18 - 4.99, $\mathrm{p}=0.015$ ). 
Table 4. Bivariate Analysis of the Association between Caregiver Burden with Clinical Characteristics among the Study Subjects using Chi-square Test $(\mathrm{N}=126)$

\begin{tabular}{|c|c|c|c|c|c|c|}
\hline \multirow{3}{*}{ Characteristics } & \multicolumn{2}{|c|}{ Caregiver Burden ${ }^{a}$} & \multirow[t]{3}{*}{$\chi^{2}$} & \multirow[t]{3}{*}{ OR } & \multirow[t]{3}{*}{$95 \% \mathrm{CI}$} & \multirow[t]{3}{*}{ p-value } \\
\hline & No & Yes & & & & \\
\hline & $\mathrm{n}(\%)$ & $\mathrm{n}(\%)$ & & & & \\
\hline Severe cancer stage in relative ${ }^{b}$ & & & 2.610 & 1.88 & $0.87-4.08$ & 0.106 \\
\hline No & $42(49.4)$ & $43(50.6)$ & & & & \\
\hline Yes & $14(34.1)$ & $27(65.9)$ & & & & \\
\hline Treatment setting of relative & & & 4.725 & 2.21 & $1.08-4.55$ & $0.030^{*}$ \\
\hline Out-patient & $30(55.6)$ & $24(44.4)$ & & & & \\
\hline In-patient & $26(36.1)$ & $46(63.9)$ & & & & \\
\hline Relative on active treatment & & & 0.002 & 1.02 & $0.46-2.25$ & 0.964 \\
\hline Yes & $41(44.6)$ & $51(55.4)$ & & & & \\
\hline No & $15(44.1)$ & $19(55.9)$ & & & & \\
\hline Relative on palliative treatment & & & 0.918 & 0.51 & $0.13-2.07$ & 0.338 \\
\hline Yes & $3(30.0)$ & $7(70.0)$ & & & & \\
\hline No & $53(45.7)$ & $63(54.3)$ & & & & \\
\hline Relative able to work $^{\mathrm{c}}$ & & & 5.930 & 2.43 & $1.18-4.99$ & $0.015^{*}$ \\
\hline Yes & $33(55.9)$ & $26(44.1)$ & & & & \\
\hline No & $23(34.3)$ & $44(65.7)$ & & & & \\
\hline Relative capable of self-care ${ }^{d}$ & & & 2.978 & 2.55 & $0.86-7.58$ & 0.084 \\
\hline Yes & $51(47.7)$ & $56(52.3)$ & & & & \\
\hline No & $5(26.3)$ & $14(73.7)$ & & & & \\
\hline Shared caregiving & & & 1.718 & 0.55 & $0.23-1.35$ & 0.190 \\
\hline No & $9(33.3)$ & $18(66.7)$ & & & & \\
\hline Yes & $47(47.5)$ & $52(52.5)$ & & & & \\
\hline Caregiving duration (months) & & & 0.129 & 0.88 & $0.44-1.78$ & 0.720 \\
\hline$<9$ & $27(42.9)$ & $36(57.1)$ & & & & \\
\hline$\geq 9$ & $29(46.0)$ & $34(54.0)$ & & & & \\
\hline Caregiving hours in a week & & & 0.514 & 1.29 & $0.64-2.62$ & 0.473 \\
\hline$<128$ & $30(47.6)$ & $33(52.4)$ & & & & \\
\hline$\geq 128$ & $26(41.3)$ & $37(58.7)$ & & & & \\
\hline
\end{tabular}

$\chi^{2}$, Chi-square; OR, Odds ratio; CI, Confidence interval; * Statistically significant $(\mathrm{p}<0.05) ;{ }^{\text {a }}$ Presence of caregiver burden was based on the total MZBI score of equal or more than $22 ;{ }^{b}$ Severe cancer stage was referring to stage 4 cancer; ${ }^{c}$ Ability to work was based on the ECOG score of 1 or below; ${ }^{\mathrm{C}}$ Capacity for self-care was based on the ECOG score of 2 or below.

\section{Association between Caregiver Burden and Depression,} Religiosity and Religious Coping

The associations between caregiver burden and their levels of depression, religiosity, and religious coping were tested using Chi-square test (Table 5). A significant positive association was found between caregiver burden and caregiver depression (OR 5.20, CI $2.41-11.21$, p < 0.001). This finding can be interpreted in two possible ways: depressed caregivers are more likely to have a significant caregiver burden than non-depressed caregivers, or burdened caregivers are more likely to be depressed than non-burdened caregivers. Caregivers with high negative religious coping (NRC) also experienced significantly more burden than those with low NRC (OR 2.21, CI 1.08 $-4.55, p=0.03)$. However, no significant relationship was found between caregiver burden with the religiosity level or positive religious coping pattern of the caregiver.
Factors Associated with Caregiver Burden: A Multivariate Analysis

Significant factors from the bivariate analyses were included in the multiple logistic regression analysis to determine the associated factor (s) for caregiver burden after controlling for the confounders (Table 6). According to this regression model, the caregivers' relationship of being the offspring of cancer patients (OR 0.41, CI 0.18 $-0.94, p=0.035)$, as well as the presence of caregiver depression (OR 4.26, CI 1.87-9.72, $\mathrm{p}=0.001$ ) remained significantly associated with caregiver burden.

In other words, the odds of having significant caregiver burden in caregivers who were the offspring of cancer patients were 2.44 times less than the odds in non-offspring caregivers in this study. Also, the presence of depression in the caregivers increased the odds of having significant caregiver burden up to four-folds.

With five significant variables, this logistic regression 
Table 5. Bivariate Analysis of the Association between Caregiver Burden with Depression, Religiosity, and Religious Coping among the Study Subjects using Chi-square Test $(\mathrm{N}=126)$

\begin{tabular}{|c|c|c|c|c|c|c|}
\hline \multirow{3}{*}{ Characteristics } & \multicolumn{2}{|c|}{ Caregiver Burden ${ }^{\text {a }}$} & \multirow[t]{3}{*}{$\chi^{2}$} & \multirow[t]{3}{*}{ OR } & \multirow[t]{3}{*}{$95 \% \mathrm{CI}$} & \multirow[t]{3}{*}{ p-value } \\
\hline & No & Yes & & & & \\
\hline & $\mathrm{n}(\%)$ & $\mathrm{n}(\%)$ & & & & \\
\hline Depression $^{\mathrm{b}}$ & & & 18.744 & 5.20 & $2.41-11.21$ & $<0.001^{*}$ \\
\hline No & $35(67.3)$ & $17(32.7)$ & & & & \\
\hline Yes & $21(28.4)$ & $53(71.6)$ & & & & \\
\hline Religiosity level $^{\mathrm{c}}$ & & & 0.040 & 1.08 & $0.53-2.18$ & 0.841 \\
\hline Low & $25(45.5)$ & $30(54.5)$ & & & & \\
\hline High & $31(43.7)$ & $40(56.3)$ & & & & \\
\hline Positive religious coping ${ }^{d}$ & & & 0.637 & 0.75 & $0.37-1.52$ & 0.425 \\
\hline Low & $24(40.7)$ & $35(59.3)$ & & & & \\
\hline High & $32(47.8)$ & $35(52.2)$ & & & & \\
\hline Negative religious coping ${ }^{\mathrm{e}}$ & & & 4.725 & 2.21 & $1.08-4.55$ & $0.030^{*}$ \\
\hline Low & $30(55.6)$ & $24(44.4)$ & & & & \\
\hline High & $26(36.1)$ & $46(63.9)$ & & & & \\
\hline
\end{tabular}

Table 6. Multivariate Analysis of the Factors Associated with Caregiver Burden among the Study Subjects using Multiple Logistic Regression Method $(\mathrm{N}=126)$

\begin{tabular}{|c|c|c|c|c|}
\hline Characteristics & Crude OR & Adjusted OR & 95\% CI (Adjusted OR) & p-value \\
\hline \multicolumn{5}{|l|}{ Relationship to the cancer patient } \\
\hline Non-children/grandchildren & 0.46 & 0.41 & $0.18-0.94$ & 0.035 \\
\hline \multicolumn{5}{|l|}{ Children/grandchildren } \\
\hline \multicolumn{5}{|l|}{ Treatment setting of relative } \\
\hline Out-patient & 2.21 & 1.10 & $0.46-2.66$ & 0.834 \\
\hline \multicolumn{5}{|l|}{ In-patient } \\
\hline \multicolumn{5}{|l|}{ Relative able to work ${ }^{a}$} \\
\hline Yes & 2.43 & 2.02 & $0.84-4.85$ & 0.115 \\
\hline \multicolumn{5}{|l|}{ No } \\
\hline \multicolumn{5}{|l|}{ Depression $^{\mathrm{b}}$} \\
\hline No & 5.20 & 4.26 & $1.87-9.72$ & 0.001 \\
\hline \multicolumn{5}{|l|}{ Yes } \\
\hline \multicolumn{5}{|l|}{ Negative religious coping ${ }^{c}$} \\
\hline Low & 2.21 & 2.04 & $0.91-4.58$ & 0.085 \\
\hline High & & & & \\
\hline
\end{tabular}

OR, Odds ratio; CI, Confidence interval; ${ }^{\mathrm{a}} \mathrm{n}$ Ability to work was based on the ECOG score of 1 or below; ${ }^{\mathrm{b}}$ Presence of caregiver depression was based on the MCES-D score of equal or more than 16; ${ }^{c}$ Negative religious coping level was determined based on total M-RCOPE NRC cut-off score of 10 (median).

model explained 27.9\% (Nagelkerke R2 $=0.279$ ) of the variance on caregiver burden and correctly classified $73 \%$ of the caregivers. The model fits well to the data with good calibration, as suggested by the Hosmer-Lemeshow goodness-of-fit test that yielded a $\chi^{2}(8)$ of 3.599 and was insignificant $(\mathrm{p}=0.891)$.

\section{Discussion}

Caregiver Burden Rate

The caregiver burden rate among family cancer caregivers in this study was estimated at $55.6 \%$, which is lower than the rates (ranged from 67.3 to $75 \%$ ) reported from studies conducted in the United States [19-33], but much higher than the rate $(24.6 \%)$ reported in another local study conducted in Kuala Lumpur [10].

The difference in rates may be explained by the distinct caregiver demographics from each study site, as well as the use of non-standardized caregiver burden assessment tools in these studies. For instance, Depression, Anxiety, Stress Scale (DASS) was used by Mahadevan et al. [10], while Hsu et al. [19] employed the Caregiver Strain Index 
(CSI). These scales have different scoring systems from the ZBI, thus producing vast difference in the burden rates. There was also no uniform criteria or definition on caregiver burden across the studies. An example is the use of 'caregiving stress' instead of 'caregiver burden' [10].

A more appropriate comparison of the caregiver burden rate is a similar cross-sectional, hospital-based study conducted among 200 family caregivers of cancer patients from India, using a validated Hindi version of ZBI [34]. The caregiver burden rate reported in this study was $43.5 \%$, a much closer value to the rate found in the current study.

In summary, the high caregiver burden rate found in the present study indicates that cancer caregiving is a challenging task. The high rate may be explained by the vulnerabilities faced by the local caregivers, who were mostly of lower socioeconomic status, and was less equipped with the knowledge and support in caregiving compared to the caregivers from developed countries. Furthermore, the local healthcare systems often neglect the needs of the caregivers and do little to assist them in looking after their welfares [21].

\section{Factors Associated with Caregiver Burden}

Among the various sociodemographic factors examined, caregiver's relationship as the children or grandchildren of cancer patients was the only factor found to be significantly associated with caregiver burden. This factor remained significant after controlling for the confounding variables in multivariate analysis. The level of significance was not observed however when examining the association between spousal caregiving and caregiver burden.

According to Wolff and Kasper [35], nearly $80 \%$ of the informal caregivers who care for an older adult are spouses or adult children, as being demonstrated in the present study where the spouses and the offspring constituted $85.9 \%$ of the total caregivers. Spouses are usually the first to provide care (primary caregiver) to an older adult who is sick. Adult children generally function as the secondary caregiver and only step into the main caregiving roles when spouses are not available [36-37].

The caregiver's relationship to cancer patients was found to be related to caregiver burden [8-18-36]. Most studies reported that spousal caregivers have a greater burden than adult children caregivers. A notable exception is a study by Given et al. [18] who reported the opposite where adult children caregivers were found to have high levels of depressive symptoms and feeling of abandonment (a portion of caregiver burden) when caring for terminal cancer patients.

In the present study, it was discovered that offspring cancer caregivers were significantly less likely to experience caregiver burden than non-offspring caregivers. However, it is interesting to note that spousal caregivers were not found to be significantly experiencing more burden than non-spousal caregivers as reported in earlier studies. The lack of significant association between spousal caregiving and caregiver burden in this study may be due to the relatively small sample size and the use of non-random sampling method.

There are a few possible explanations on why adult children who care for cancer patients may experience fewer burden than other caregiver groups. Firstly, as mentioned before, the majority of the offspring caregivers are secondary caregivers as compared to the spouses who are the primary caregivers. As the children only involve in assisting the primary caregiver, the caregiving expectations and workloads are understandably lower in the former. Thus, adult children are generally less vulnerable to the physical and psychological distress of caregiving [37].

Secondly, the nuclearization of families following rapid urbanization of Asian societies might influence the perceived burden by the adult children caregivers. A nuclear family usually comprises of the married couple staying together with or without their dependent children. Relevant to the increasing work requirements, many adult children live separately from their parents to form a nuclear family of their own. As a result, they are only able to provide distance caregiving when a parent becomes ill, relying much on the help of other family members or formal nursing services [38].

Consequently, distance adult children caregivers with adequate social support may experience less direct burden on caregiving. Lowenstein and Gilbar [36] demonstrated that children caregivers who stayed in separate households from the cancer patients were found to experience significantly fewer burden than the spouses who resided together with their ill partners. Nevertheless, distance adult offspring caregivers may still expose to psychological distress, especially when they lack social support, as they often have multiple commitments such as child-rearing, domestic chores, full-time employment and various financial responsibilities [38].

Thirdly, the concept of filial piety, a cultural belief that influences adult children's attitudes and behaviors toward their parents, may play an important role in the buffering of care burden in adult children caregivers. In many Asian cultures, Malaysian included, children are often expected to care for their parents in their aging years, more so when the elderly parents are suffering from chronic medical illnesses [39].

Various studies demonstrated the positive effects of filial piety on the caregiving appraisal and subsequently on the caregiver burden [39-40]. According to Lai [39], filial piety may even serve as a protective factor against caregiver burden. Thus, offspring caregivers who identify more with filial piety are more likely to find caregiving tasks to be positive, beneficial and not burdensome [40]. This will help to reduce the subjective care burden as the caregivers have a more positive evaluation and understanding of their roles.

Presence of depression in cancer caregivers was another important factor that significantly associated with caregiver burden in this study. Consistent results were found in other caregiver studies, which support the significant relationship between these two variables [41-43]. As mentioned earlier, the significant relationship 
between caregiver burden and depression can be bi-directional. In general, caregiver depression has been viewed as a reaction or consequence of the caregiving processes [44]. Thus, many researchers established that both subjective and objective caregiver burdens, especially following long-term caregiving, might contribute to depression in the caregivers [43-44]. In other words, caregivers with high burden are more likely to be depressed, and vice versa.

However, Stommel et al. [41] put forward an interesting perspective that the opposite might be true where caregiver depression can predict the caregiver burden. Simply put, depressed caregivers are more likely to have high caregiver burden. The authors argued that depression, being a rather stable condition, would be a significant influence on the caregivers' perceptions and thus able to explain variations in the perceived burdens [41].

In addition to direct association, caregiver depression may also have an indirect influence on the caregiver burden [42]. One example is the finding by Utne et al. [45], which suggested that depression possibly mediates caregiver burden through the feeling of hope, as hopelessness is known to be an important component of depression. Nevertheless, the causal relationship between caregiver burden and depression was still not established in the present study due to the relatively small sample size and the cross-sectional design of the study.

To the best understanding of the authors, there is yet any local study done on the association between religious factors and caregiver burden. The role of religion in caregiving can be studied in a multireligious environment like Malaysia. Unexpectedly, the current study failed to demonstrate a significant relationship between religiosity and religious coping strategies with caregiver burden on a multivariate level. The results were inconsistent with previous studies reporting the association between these variables [46-47].

The present study, however, did show that high negative religious coping (NRC) use, but not positive religious coping (PRC), was associated with higher caregiver burden on a bivariate level. This finding is in concordance with the results from previous literature supporting the association between the NRC with the levels of psychological distress or mental health outcomes [47-48]. It is postulated that individuals who struggle with their religious beliefs may not be able to use their faith as a resource for coping, as they see their faith as distant and weak [48].

The relatively high religiosity level and use of PRC strategies among cancer caregivers in the present study were found to have no significant impact on the caregiver burden. These findings may be explained by the presence of multiple concomitant stressors, e.g. lower socioeconomic status, lack of caregiving resources or supports, the severity of cancer presentation, etc., which overshadow the buffering effects of religiosity and PRC. Interestingly, PRC may be associated with greater caregiver burden [46]. It was hypothesized that PRC may increase the possibility that one takes on number of or more difficult caregiving tasks out of a feeling of religious duty. Also, those who are the most burdened may be more likely to turn to religion as a way to cope [46].

In general, the findings from this study indicated that the influence of religiosity and religious coping on caregiver burden is still vague and inconsistent. However, this does not mean that religious factors have no value or positive role at all in the caregiving process. More local research is needed, preferably using a broad-spectrum and standardized measure, to address the multidimensional qualities of religiosity and religious coping.

\section{Limitations and Strengths of the Study}

There are several limitations present in this study. First, it was a cross-sectional study, which did not describe the temporal relationship between the caregiver burden and the various factors examined. Thus, causal inferences could not be made between these variables. A longitudinal prospective study may be a better design for this purpose.

Second, convenience or non-random sampling techniques used in this study would generate sampling bias, especially when the study was conducted in a single setting based in a government hospital. The sample chosen might not represent the general population of the cancer caregivers, which includes caregivers in the community as well as those in the private medical centers.

Third, a relatively smaller sample size could have contributed to the study having small effect size and wide confidence intervals, which further restricted the generalization of its findings, and the statistical power necessary to detect the complex relationships between the study variables. A study with larger sample size is needed to obtain a more robust finding.

Fourth, the study did not differentiate between the physical and the psychological components of caregiver burden, which may have resulted in different patterns of burden reported among the caregivers [19]. Also, potentially important information about the cancer caregivers and patients that might influence the caregiver burden was not included in the study questionnaires. Examples include the presence of medical comorbidities, the exact caregiving tasks performed with stricter criteria on caregiving hours, as well as whether the caregivers are staying together or separately from the patients, etc.

On the other hand, the current study demonstrated a few notable strengths as well. Despite being a single-centered study, it was conducted in a tertiary referral center for oncology services with cancer patients and caregivers originated from various parts of the state. This, together with the multiracial background of the study subjects, made up for the diversity of the samples. The cross-sectional design has its fair share of advantages, in which it allows the study to measure the prevalence of caregiver burden, and to explore multiple factors and outcomes at the same time. This helps to bring into perspective the effects of cancer caregiving and the caregiving problems relevant to the local population in a short period .

The study was also the first to examine the context of caregiver burden among the local family caregivers of multiracial background and various types of cancer patients, 
not limiting to just single-race caregivers [21], or a single type of cancer patients [10]. The findings from the present study would add to the growing literature on cancer caregiving and caregiver burden in the local setting.

In conclusion, this study confirmed that cancer caregiving is burdensome. A relatively high rate of caregiver burden was observed among the local family caregivers of cancer patients. Caregivers who are the offspring of cancer patients were experiencing significantly fewer burdens than non-offspring caregivers. On the other hand, caregivers who had probable depression were more likely to experience significant caregiver burden.

\section{Acknowledgments}

The authors would like to thank Dr. Yu Kong Leong, head of the oncology department and Dr. Chin Zin Hing, hospital director of Sarawak General Hospital for their approval and assistance of this research study. This research was not funded. The authors declare no conflict of interest.

\section{References}

1. Ferlay J., Colombet, M., Soerjomataram, I., Mathers, C., Parkin, D.M., Pineros, M., et al. Estimating the global cancer incidence and mortality in 2018: GLOBOCAN sources and methods. Int. J. Cancer. 2019;144(8):1941-53.

2. Manan, A.A., Tamin, N.S.I., Abdullah, N.H., Abidin, A.Z., \& Wahab, M. Malaysian national cancer registry report 2007 - 2011. Putrajaya: National Cancer Institute, Ministry of Health Malaysia; 2016.

3. Stein, K.D., Syrjala, K.L., \& Andrykowski, M.A. Physical and psychological long-term and late effects of cancer. Cancer. 2008;112(11):2577-92.

4. Silver, J.K., Baima, J., \& Mayer, R.S. Impairment-driven cancer rehabilitation: an essential component of quality care and survivorship. CA: A Cancer Journal for Clinicians. 2013;63:295-317.

5. Lim, S.M., Kim, H.C., \& Lee, S. Psychosocial impact of cancer patients on their family members. Cancer Res. Treat. 2013;45(3):226-33.

6. Wozniak, K., \& Izycki, D. Cancer: a family at risk. Prz Menopauzalny. 2014;13(4):253-61.

7. Family Caregiver Alliance [Internet]. Definitions. 2014 Jan 31 [cited 2016 August 24]. Available from: https://www. caregiver.org/definitions-0

8. Hagedoorn, M., Sanderman, R., Bolks, H.N., Tuinstra, J., \& Coyne, J.C. Distress in couples coping with cancer: A meta-analysis and critical review of role and gender effects. Psychol. Bull. 2008;134(1):1-30.

9. Northouse, L.L., Katapodi, M.C., Schafenacker, A.M., \& Weiss, D. The impact of caregiving on the psychological well-being of family caregivers and cancer patients. Semin. Oncol. Nurs. 2012;28(4):236-45.

10. Mahadevan, R., Jaafar, N.R.N., Din, S.H.S., Ahmad, S.N.A., Baharuddin, A., \& Razali, R. The stress of caregiving: A study of family caregivers of breast cancer patients receiving oncologic treatment at a Malaysian general hospital. Sains Malaysiana, 2013;42(7):1019-26.

11. Payne, S., Smith, P., \& Dean, S. Identifying the concerns of informal carers in palliative care. Palliat. Med. 1999;13(1):37-44.
12. Shekarian, T., Valsesia-Wittmann, S., Caux, C., \& Marabelle, A. Paradigm shift in oncology: targeting the immune system rather than cancer cells. Mutagenesis. 2015;30(2):205-11.

13. Nijboer, C., Tempelaar, R., Sanderman, R., Triemstra, M., Spruijt, R.J., \& van den Bos, G.A. Cancer and caregiving: the impact on the caregiver's health. Psycho-Oncol. 1998;7(1):3-13

14. Girgis, A., Lambert, S., Johnson, C., Waller, A., \& Currow, D. Physical, psychosocial, relationship, and economic burden of caring for people with cancer: a review. J. Oncol. Pract. 2013;9(4):197-202.

15. Stenberg, U., Ruland, C.M., \& Miaskowski, C. Review of the literature on the effects of caring for a patient with cancer. Psycho-Oncol. 2010;19(10):1013-25.

16. Ano, G.G., \& Vasconcelles, E.B. Religious coping and psychological adjustment to stress: a meta-analysis. J. Clin. Psychol. 2005;6(14):461-80.

17. Weaver, A.J., \& Flannelly, K.J. The role of religion/ spirituality for cancer patients and their caregivers. South. Med. J. 2004;97(12):1210-14

18. Given, B., Wyatt, G., Given, C., Sherwood, P., Gift, A., DeVoss, D., et al. Burden and depression among caregivers of patients with cancer at the end of life. Oncol. Nurs. Forum. 2004;31(6):1105-15.

19. Hsu, T., Loscalzo, M., Ramani, R., Forman, S., Popplewell, L., Clark, K., et al. Factors associated with high burden in caregivers of older adults with cancer. Cancer. 2014;120(18):2927-35.

20. Jaafar, N.R.N., Din, S.H.S., Saini, S.M., Ahmad, S.N.A., Midin, M., Sidi, H., et al. Clinical depression while caring for loved ones with breast cancer. Compr. Psychiatry. 2014;55:S52-9

21. Cheong, R., \& Putit, Z. A qualitative study: Chinese cancer caregivers in Sarawak. Journal of Malaysian Nurses Association [Internet]. 2011 Jan [cited 2017 June 10]. Available from: https://www.researchgate.net/ publication/283635915 Rina C and Zabidah P 2011 A Qualitative_study_Chinese_Cancer_Caregivers_in Sarawak Journal_of_Malaysian_Nurses_Association

22. Shim, V.K., Ng, C.G., \& Drahman, I. Validation of the Malay version of Zarit Burden Interview (MZBI). Malaysian Journal of Psychiatry. 2017;26(2):3-18.

23. Hébert, R., Bravo, G., \& Préville, M. Reliability, validity and reference values of the Zarit Burden Interview for assessing informal caregivers of community-dwelling older persons with dementia. Canadian Journal on Aging. 2000;19(4):494-507.

24. Zarit, S.H., Orr, N.K., \& Zarit, J.M. The hidden victims of Alzheimer's disease: families under stress. New York: New York University Press; 1985.

25. Radloff, L.S. The CES-D scale: a self-report depression scale for research in the general population. Appl. Psychol. Meas. 1977;1:385-401.

26. Sabki, Z.A., Zainal, N.Z., \& Ng, C.G. Exploratory factorial analysis and reliability of the Malay version of Centre for Epidemiological Studies - Depression scale (MCES-D) in a group of Malaysian junior doctors. Int. J. Acad. Res. 2014;6(2):333-7.

27. Koenig, H.G., Parkerson, G.R., \& Meador, K.G. Religion index for psychiatric research: a 5-item measure for the use in health outcome studies. Am. J. Psychiatry. 1997;154(6):88597.

28. Koenig, H.G., \& Büssing, A. The Duke University Religion Index (DUREL): a five-item measure for use in epidemiological studies. Religions. 2010;1:78-85.

29. Nurasikin, M.S., Aini, A., Aida, S.A.A. \& Ng, C.G. Validity and reliability of the Malay version of Duke University 
Religion Index (DUREL-M) among a group of nursing student. Malaysian Journal of Psychiatry. 2010;9(2):68-72.

30. Pargament, K., Feuille, M., \& Burdzy, D. The Brief RCOPE: current psychometric status of a short measure of religious coping. Religions. 2011;2(1)51-76.

31. Yusoff, N., Low, W.Y., \& Yip, C.H. Reliability and validity of the Malay version of BriefCOPE scale: a study on Malaysian women treated with adjuvant chemotherapy for breast cancer. Malaysian Journal of Psychiatry. 2009;18:27-35.

32. Oken, M.M., Creech, R.H., Tormey, D.C., Horton, J., Davis, T.E., McFadden, E.T., et al. Toxicity and response criteria of the Eastern Cooperative Oncology Group. Am. J. Clin. Oncol. 1982;5(6):649-55.

33. Kim, Y., \& Schulz, R. Family caregivers' strain: comparative analysis of cancer caregiving with dementia, diabetes, and frail elderly caregiving. J. Aging Health. 2008;20(5):483503.

34. Lukhmana, S., Bhasin, S.K., Chhabra, P., \& Bhatia, M.S. Family caregivers' burden: a hospital based study in 2010 among cancer patients from Delhi. Indian J. Cancer. 2015;52(1):146-51.

35. Wolff, J.L., \& Kasper, J.D. Caregivers of frail elders: updating a national profile. Gerontologist. 2006;46:344-56.

36. Lowenstein, A., \& Gilbar, O. The perception of caregiving burden on the part of elderly cancer patients, spouses and adult children. Families, Systems \& Health. 2000;18(3):33746.

37. Revenson, T.A., Griva, K., Luszczynska, A., Morrison, V., Panagopoulou, E., Vilchinsky, N., et al. Caregiving in the illness context. Hampshie, England: Palgrave Macmillan; 2015.

38. Mazanec, P. Distance caregiving a parent with cancer. Semin. Oncol. Nurs. 2012;28(4):271-8.

39. Lai, D.W.L. Filial piety, caregiving appraisal, and caregiving burden. Research on Aging. 2010;32(2):200-23.

40. Khalaila, R., \& Litwin, H. Does filial piety decrease depression among family caregivers? Aging Ment. Health. 2011;15(6):679-86.

41. Stommel, M., Given, C.W., \& Given, B. Depression as an overriding variable explaining caregiver burdens. J. Aging Health. 1990;2(1):81-102.

42. Grov, E.K., Fossa, S.D., Sorebo, O., \& Dahl, A.A. Primary caregivers of cancer patients in the palliative phase: a path analysis of variables influencing their burden. Soc. Sci. Med. 2006;63(9): 2429-2439.

43. Rivera, H.R. Depression symptoms in cancer caregivers. Clin. J. Oncol. Nurs. 2009;13(2):195-202.

44. Rhee, Y.S., Yun, Y.H., Park, S., Shin, D.O., Lee, K.M., Yoo, J.H., et al. Depression in family caregivers of cancer patients: the feeling of burden as a predictor of depression. J. Clin. Oncol. 2008;26(36):5890-5.

45. Utne, I., Miaskowski, C., Paul, S.M., \& Rustøen, T. Association between hope and burden reported by family caregivers of patients with advanced cancer. Support Care Cancer. 2013;21:2527-35.

46. Pearce, M.J., Singer, J.L., \& Prigerson, H.G. Religious coping among caregivers of terminally ill cancer patients: main effects and psychosocial mediators. J. Health Psychol. 2006;11(5):743-59.

47. Herrera, A.P., Lee, J.W., Nanyonjo, R.D., Laufman, L.E., \& Torres-Vigil, I. Religious coping and caregiver wellbeing in Mexican-American families. Aging Ment. Health. 2009;13(1):84-91.

48. Ng, G.C., Mohamed, S., Sulaiman, A.H., \& Zainal, N.Z. Anxiety and depression in cancer patients: the association with religiosity and religious coping. J. Religion Health, 2016;56(2):575-90.
This work is licensed under a Creative Commons AttributionNon Commercial 4.0 International License. 